\title{
Organ Dose Conversion Coefficients Calculated for Korean Pediatric and Adult Voxel Phantoms Exposed to External Photon Fields
}

\author{
Choonsik Lee', Yeon Soo Yeom¹', Keith Griffin'1, Choonik Lee², Ae-Kyoung Lee ${ }^{3}$, Hyung-do Choi ${ }^{3}$ \\ ${ }^{1}$ Division of Cancer Epidemiology and Genetics, National Cancer Institute, National Institutes of Health, Rockville, MD, USA; ${ }^{2}$ Department of Radiation \\ Oncology, University of Michigan, Ann Arbor, MI, USA; ${ }^{3}$ Electronics and Telecommunications Research Institute, Daejeon, Korea
}

\section{Invited Paper}

Received February 20, 2020

Revision March 21, 2020

Accepted May 4, 2020

Corresponding author: Choonsik Lee

Division of Cancer Epidemiology and Genetics, National Cancer Institute, National Institutes of Health, 9609 Medical Center Dr, Rockville, MD, USA

E-mail: choonsik.lee@nih.gov

\begin{abstract}
This is an open-access article distributed under the terms of the Creative Commons Attribution License (http://creativecommons.org/licenses/by-nc/4.0), which permits unrestricted use, distribution, and reproduction in any medium, provided the original work is properly cited.
\end{abstract}

Copyright $\odot 2020$ The Korean Association for Radiation Protection

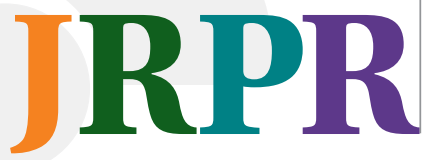

Background: Dose conversion coefficients (DCCs) have been commonly used to estimate radiation-dose absorption by human organs based on physical measurements of fluence or kerma. The International Commission on Radiological Protection (ICRP) has reported a library of DCCs, but few studies have been conducted on their applicability to non-Caucasian populations. In the present study, we collected a total of 8 Korean pediatric and adult voxel phantoms to calculate the organ DCCs for idealized external photon-irradiation geometries.

Materials and Methods: We adopted one pediatric female phantom (ETRI Child), two adult female phantoms (KORWOMAN and HDRK Female), and five adult male phantoms (KORMAN, ETRI Man, KTMAN1, KTMAN2, and HDRK Man). A general-purpose Monte Carlo radiation transport code, MCNPX2.7 (Monte Carlo N-Particle Transport extended version 2.7), was employed to calculate the DCCs for 13 major radiosensitive organs in six irradiation geometries (anteroposterior, posteroanterior, right lateral, left lateral, rotational, and isotropic) and 33 photon energy bins $(0.01-20 \mathrm{MeV})$.

Results and Discussion: The DCCs for major radiosensitive organs (e.g., lungs and colon) in anteroposterior geometry agreed reasonably well across the 8 Korean phantoms, whereas those for deep-seated organs (e.g., gonads) varied significantly. The DCCs of the child phantom were greater than those of the adult phantoms. A comparison with the ICRP Publication 116 data showed reasonable agreements with the Korean phantom-based data. The variations in organ DCCs were well explained using the distribution of organ depths from the phantom surface.

Conclusion: A library of dose conversion coefficients for major radiosensitive organs in a series of pediatric and adult Korean voxel phantoms was established and compared with the reference data from the ICRP. This comparison showed that our Korean phantom-based data agrees reasonably with the ICRP reference data.

Keywords: Korean Voxel Phantoms, Monte Carlo Radiation Transport, Organ Dose Conversion Coefficients, External Photon Beams

\section{Introduction}

Dose conversion coefficients (DCCs) have been commonly used to estimate radiation dose absorbed in human organs from physical measurements of fluence or kerma at the given radiation field in the air since the direct measurement of organ doses is technically and practically challenging [1]. The DCCs are derived from computer simu- 
lations based on Monte Carlo radiation transport methods combined with computerized human anatomy, called computational human phantoms. The phantoms have evolved from stylized phantoms simplifying the human anatomy using mathematical equations to voxel phantoms based on tomography images of the human body. Following the evolution, the International Commission on Radiological Protection (ICRP) reported a library of DCCs in different steps: ICRP Publication 74 [2] based on stylized phantoms and ICRP Publication 116 [1] using more advanced voxel phantoms [3]. Both the stylized and voxel phantoms used in those publications were developed to match the ICRP reference data representing Caucasian population. Few studies were conducted on their applicability to non-Caucasian populations where only a small number of Korean or Japanese voxel phantoms were used for comparison with ICRP Publication $74[4,5]$.

A series of Korean computational phantoms have been reported for use in ionizing and non-ionizing radiation dosimetry. The first Korean adult male and female voxel phantoms, called Korean Man (KORMAN) [4] and Korean Woman (KORWOMAN) [5], were constructed based on magnetic resonance (MR) images. The same research group subsequently reported more detailed phantoms of two Korean adult males, Korean Typical Man 1 (KTMAN1) and Korean Typical Man 2 (KTMAN2), constructed from high-resolution MR and computed tomography (CT) images, respectively [6]. More recently, another Korean research group reported Korean adult male and female voxel phantoms, High-Definition Reference Korean man (HDRK Man) [7] and High-Definition Reference Korean woman (HDRK Woman) [8], constructed from high-resolution color slice images of Korean adult cadavers acquired from the Visible Korean Human (VKH) project [9]. In contrast to other Korean voxel phantoms, the height, weight, and organ/tissue mass of the HDRK Man and HDRK Woman were adjusted to match the Korean reference data [10]. While these Korean voxel phantoms listed above were developed for ionizing radiation dosimetry, a research group at the Electronics and Telecommunications Research Institute (ETRI) constructed a 21-year-old adult male voxel phantom [11] (denoted as ETRI Man in this paper), and a 7-year-old child female voxel phantom [12] (denoted as ETRI Child) based on MR images for dosimetry applications in non-ionizing radiation exposures.

In the present study, we collected a total of 8 Korean voxel phantoms ( 1 pediatric, 2 adult females, and 5 adult males) mentioned above to calculate a library of organ DCCs for idealized external photon irradiation geometries. The results were then compared with those from ICRP Publication 116, the latest data calculated from the ICRP reference adult male and female voxel phantoms [3].

\section{Materials and Methods}

\section{Korean Computational Phantoms}

We adopted a series of Korean pediatric and adult computational human phantoms listed above: 1 pediatric phantom (ETRI Child), 2 adult female phantoms (KORWOMAN and HDRK Woman), 5 adult male phantoms (KORMAN, ETRI Man, KTMAN1, KTMAN2, and HDRK Man). Major characteristics of the Korean voxel phantoms are tabulated in Table 1. Fig. 1 shows the frontal projection views of the 8 phantoms where anatomical structures were made semi-transparent.

\section{Monte Carlo Calculations of DCCs}

A general-purpose Monte Carlo radiation transport code, MCNPX2.7, was adopted for the simulation of computational human phantoms exposed to external photon beams. The original binary matrix files of the phantoms were converted to "lattice" files which were importable to MCNPX. Elemental composition and density of different organs and tissues were also implemented into MCNPX input files. Although

Table 1. Characteristics of the Eight Korean Voxel Computational Human Phantoms Adopted in the Current Study

\begin{tabular}{lccccccc}
\hline Phantom name & Age $(\mathrm{yr})$ & Sex & Height $(\mathrm{cm})$ & Weight $(\mathrm{kg})$ & Voxel resolution $(\mathrm{cm})$ & Voxel volume $\left(\mathrm{cm}^{3}\right)$ & Voxel array size \\
\hline ETRI Child & 7 & $\mathrm{~F}$ & 122 & 26 & $0.1,0.1,0.3$ & 0.003 & $372 \times 229 \times 408$ \\
KORWOMAN & 25 & $\mathrm{~F}$ & 160 & 55 & $0.2,0.2,0.8$ & 0.128 & $300 \times 150 \times 200$ \\
HDRK Woman & 26 & $\mathrm{~F}$ & 161 & 54 & $0.204,0.204,0.207$ & 0.0086 & $261 \times 109 \times 825$ \\
KORMAN & 30 & $\mathrm{M}$ & 170 & 68 & $0.2,0.2,1.0$ & 0.04 & $250 \times 120 \times 170$ \\
ETRI Man & 21 & $\mathrm{M}$ & 176 & 67 & $0.3,0.3,0.3$ & 0.027 & $167 \times 87 \times 613$ \\
KTMAN1 & 25 & $\mathrm{M}$ & 172 & 65 & $0.2,0.2,0.5$ & 0.02 & $300 \times 150 \times 344$ \\
KTMAN2 & 35 & $\mathrm{M}$ & 172 & 68 & $0.2,0.2,0.5$ & 0.02 & $300 \times 150 \times 344$ \\
HDRK Man & 33 & $\mathrm{M}$ & 171 & 68 & $0.198,0.198,0.209$ & 0.0082 & $247 \times 141 \times 850$ \\
\hline
\end{tabular}



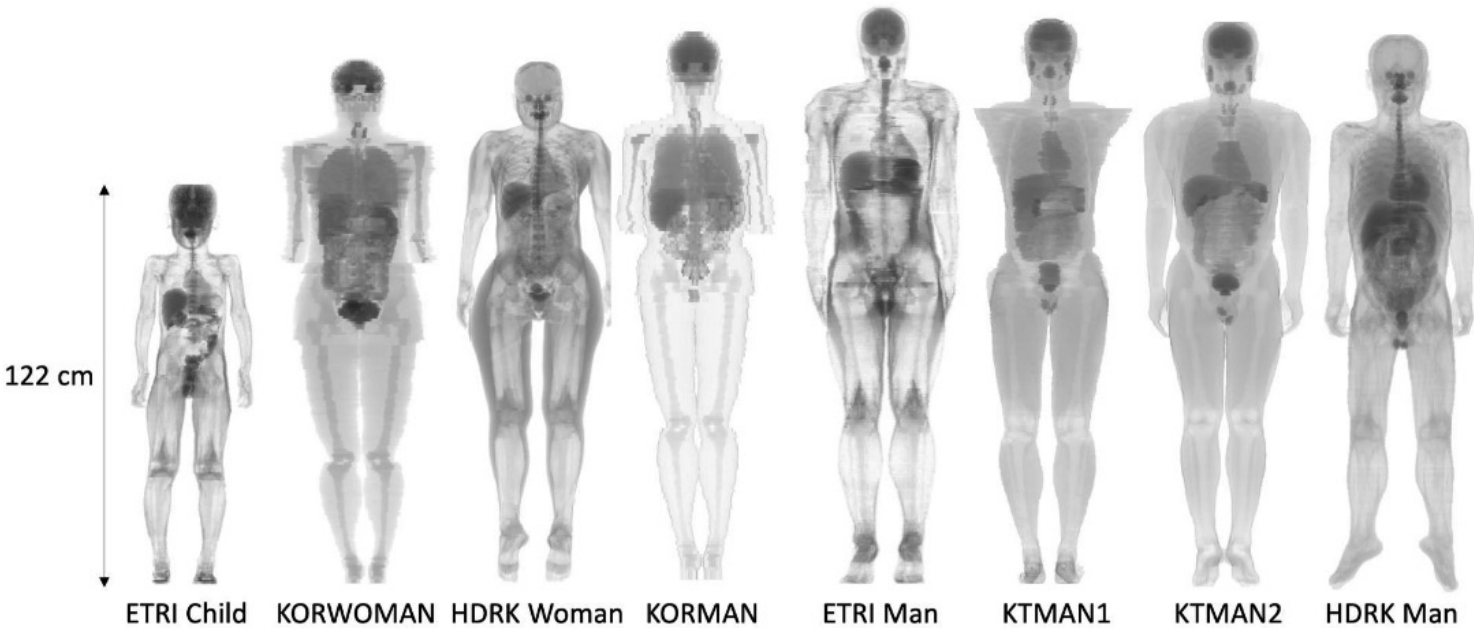

Fig. 1. Frontal projection views of the one pediatric (ETRI Child), two adult females (KORWOMAN and HDRK Woman), and five adult males (KORMAN, ETRI Man, KTMAN1, KTMAN2, and HDRK Man). Korean computational phantoms adopted for organ dose calculations.

the material data were not completely identical across the 8 phantoms, we confirmed that those are overall consistent with the data from ICRP Publication 89 [11]. A total of 33 photon energy bins (0.01-20 MeV) and 6 irradiation geometries were adopted from ICRP Publication 116 [1]: anteroposterior (AP), posteroanterior (PA), right lateral (RLAT), left lateral (LLAT), rotational (ROT), and isotropic (ISO). We calculated DCCs for 13 organs commonly available in all 8 phantoms: brain, thyroid, lungs (left and right combined), small intestine wall, colon wall, stomach wall, liver, kidneys (left and right combined), urinary bladder wall, heart, esophagus wall, spleen, and gonads (ovaries for the female phantoms and testes for the male phantoms, left and right combined). We did not include other organs which are not available in some phantoms. Absorbed doses (Gy) to the organs were calculated by using the F6:E tally with secondary electrons tracks included. The absorbed doses were converted to absorbed doses per unit fluence, and then to absorbed doses per unit air kerma using conversion coefficients for air kerma per unit fluence [1]. A total of 100 million photon history for each simulation was used to reduce statistical relative errors below $5 \%$ for all organ doses except for small organs (e.g., thyroid) below $10 \%$. The simulations were conducted on the high-performance computing cluster, Biowulf (http://hpc. nih.gov), installed at the National Institutes of Health.

\section{Analysis of Dose Conversion Coefficients}

We analyzed the results in different aspects. First, we compared DCCs for the lungs, colon wall, and gonads (ICRP tissue weighting factor greater than 0.05 [12]) across the Korean phantoms in different irradiation geometries to investigate how the results agree or differ. Second, we compared DCCs from the child female phantom (ETRI Child) with the mean adult DCCs averaged over the two adult female phantoms to evaluate the impact of age on DCCs. Finally, we compared our adult DCCs with those from the ICRP reference adult phantoms to assess the applicability of the Caucasian-based data to Korean adult population. In all comparisons, we selected DCCs at $0.1 \mathrm{MeV}$ since DCC generally reaches its peak around 0.06-0.1 MeV.

To better analyze the agreement or discrepancy in DCCs, we measured the distribution of organ depths from the phantom surface in different irradiation geometries using an in-house script. The process selected a random voxel within the organ of interest and selected a random point within that voxel. The script then calculated a distance from the sampled point to the phantom surface. Distance calculations were made 500,000 times per organ with a resulting distribution of depths.

\section{Results and Discussion}

A comprehensive library of organ dose conversion coefficients calculated for 13 organs in the 8 pediatric and adult phantoms exposed in the irradiation geometry of AP, PA, RLAT, LLAT, ROT, and ISO was established. The full dataset is downloadable from the journal website (Supplementary Materials).

The DCCs for the lungs (Fig. 2A) and colon (Fig. 2B) from all phantoms in AP geometry reasonably agree with the coef- 

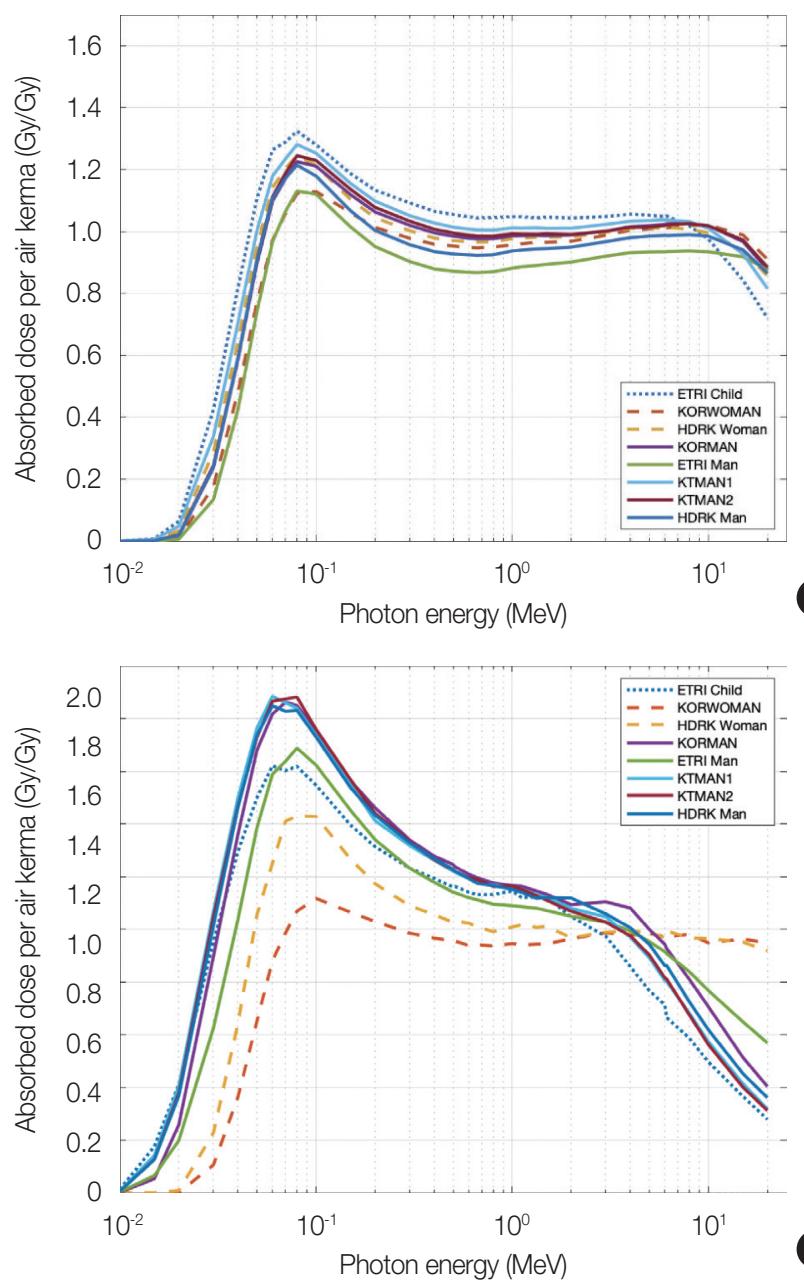

ficient of variation (COV) $5 \%$ and $3 \%$, respectively, in DCCs at $0.1 \mathrm{MeV}$. Fig. 2C shows that DCCs for the gonads (ovaries for the female phantoms and testes for the male phantoms) significantly differ across the 8 phantoms in AP geometry: COV in DCCs at $0.1 \mathrm{MeV}$ was $14 \%$. The DCCs for the testes from the 5 adult male phantoms are greater than those of the ovaries of the child and 2 adult female phantoms.

DCCs of ETRI Child female phantom were overall greater than those of the 2 adult female phantoms. As an example, DCCs for the ETRI Child liver in LLAT geometry (Fig. 3A) and the ETRI Child stomach in RLAT geometry (Fig. 3B) at 0.1 $\mathrm{MeV}$ were up to 1.9- and 1.5-fold, respectively, greater than those averaged over the 2 adult female phantoms. We selected LLAT and RLAT irradiation geometries which maximize the penetrating lengths for the liver (positioned on the right) and the stomach (positioned on the left), respectively, in both child and adult phantoms.

Comparison with the data from ICRP Publication 116, which is based on the ICRP reference adult male and female

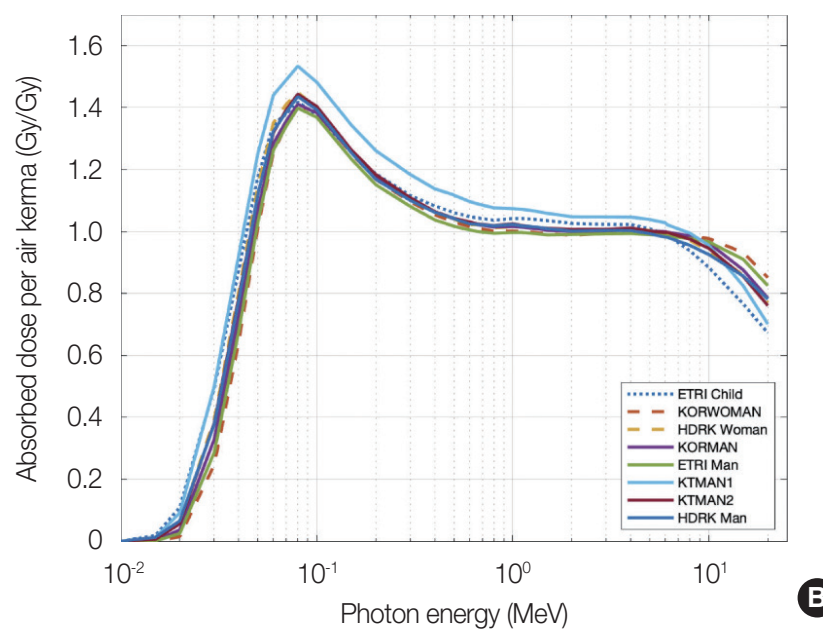

Fig. 2. Dose conversion coefficients for (A) lungs, $(B)$ colon, and $(C)$ gonads (ovaries for the female and testes for the male phantoms) for the eight Korean phantoms exposed to idealized external photons in anteroposterior (AP) geometry.

voxel phantoms showed reasonable agreements with Korean phantom-based data. The lung DCCs at $0.1 \mathrm{MeV}$ from the Korean adult phantoms differed from those of ICRP by $5 \%$ (for both male and female) (Fig. 4A). The colon DCCs at 0.1 $\mathrm{MeV}$ from the Korean adult phantoms were different from the ICRP data by less than $10 \%$ (male) and about $1 \%$ (female) (Fig. 4B).

Organ dose conversion coefficients are crucial dosimetric quantities to estimate absorbed dose to radiosensitive organs and tissues in human anatomy exposed to medical radiation procedures, natural background radiation, occupational exposure, possibly radiation accidents or terrorist attacks. To indirectly estimate organ absorbed dose for these radiological events, the ICRP has published a series of the conversion coefficients data, which however are based on the anatomical models of reference Caucasian individuals. The current study was intended to create a new organ DCC database for Korean pediatric and adult phantoms and to compare the results with the ICRP reference data. 

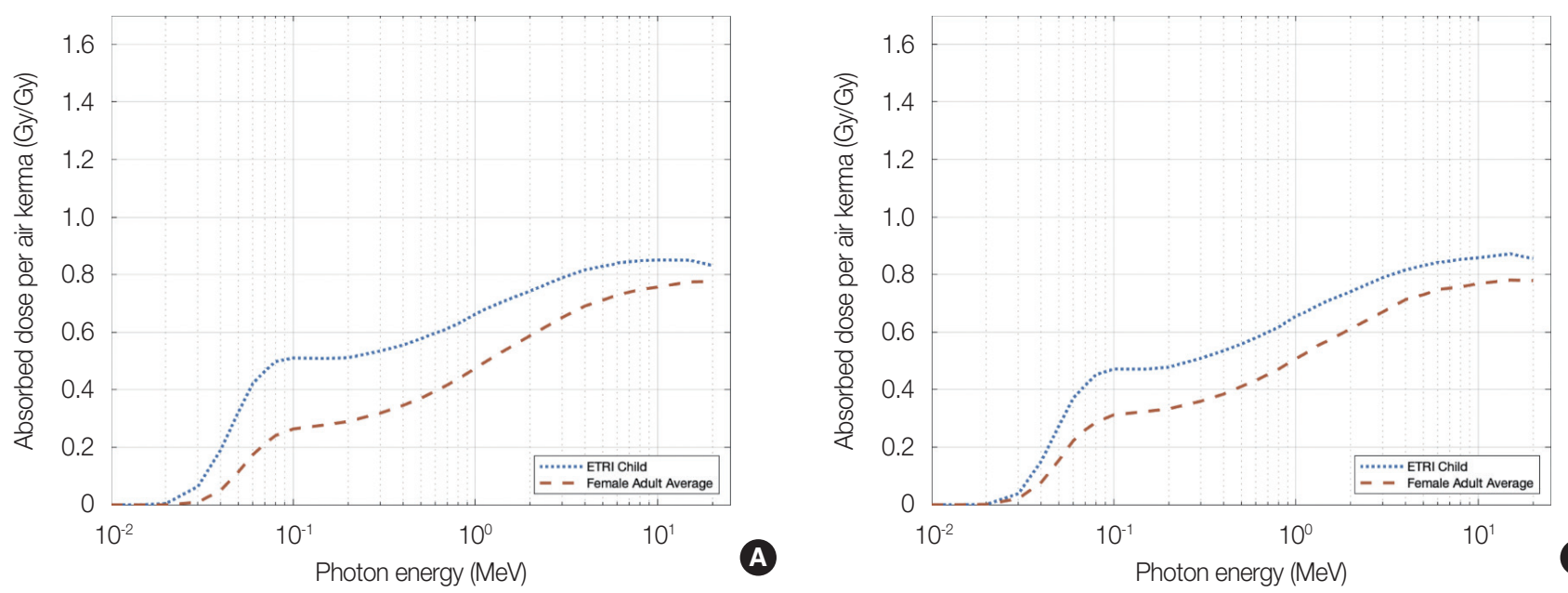

Fig. 3. Comparison of dose conversion coefficients for liver in left lateral irradiation geometry (A) and stomach in right lateral irradiation geometry (B) between ETRI Child and adult female phantom average.
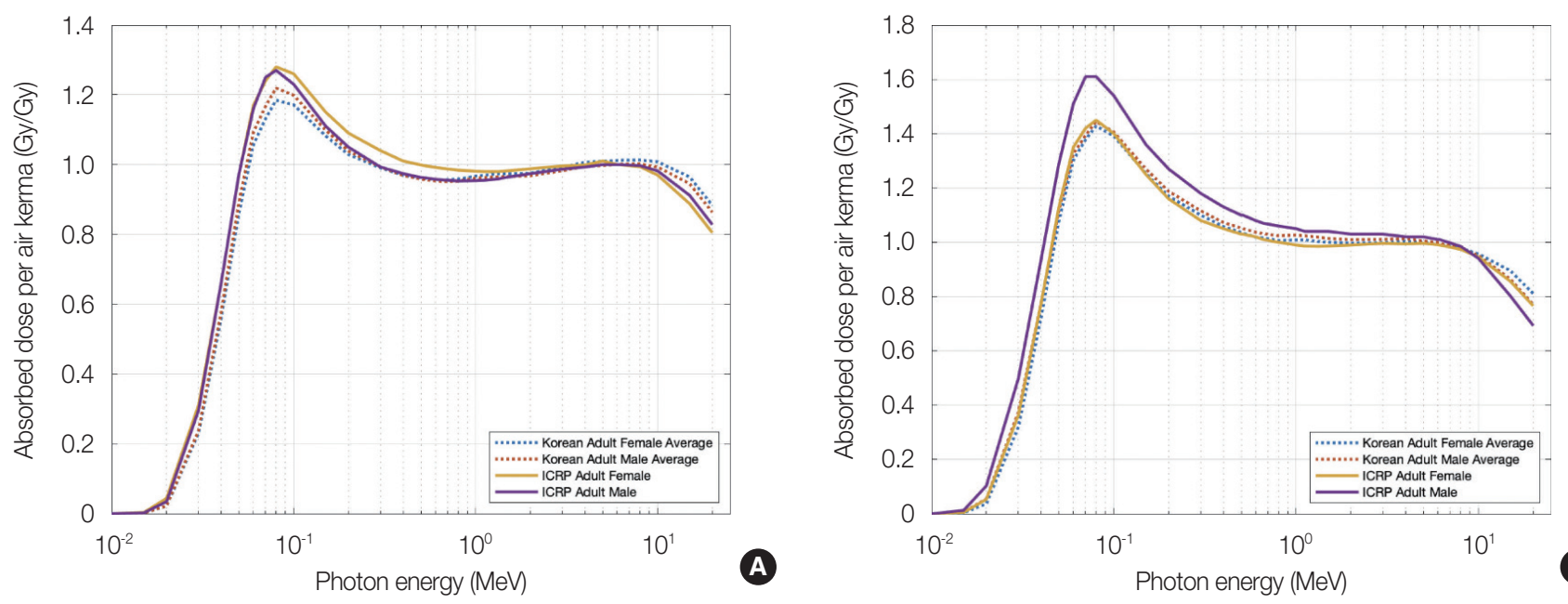

Fig. 4. Comparison of dose conversion coefficients for lungs $(A)$ and colon (B) for anteroposterior geometry between Korean adult phantoms and the International Commission on Radiological Protection (ICRP) Publication 116.

Most organs positioned in the front of the phantoms such as the lungs and colon showed agreement in AP irradiation geometry since the depth of the organs from the surface of the phantoms is relatively consistent independent of the age and gender of the phantoms. Fig. 5A shows the depth distribution of the lungs in AP geometry. All phantoms show similar depth distributions with the shift of the ETRI Child phantom, which showed the greatest DCCs compared to other phantoms (Fig. 2A). The testes and ovaries showed large variations in the depth distribution across the phantoms (Fig. 5B), resulting in significant differences in DCCs (Fig. 2C). The adult male phantoms show similar depth distribution for the testes except for the ETRI-Man phantom, where we found the testes and prostate had the same identification number. The depths for the adult female ovaries are notably shifted to the right compared to those for the adult male testes, which is reflected in the smaller DCCs in ovaries than testes (Fig. 2C). Age-dependency between the ETRI Child and adult average DCCs was observed notably in lateral irradiation geometries (Fig. 3), which was also the results of differences in organ depth between pediatric and adult groups.

Agreement between Korean adult DCCs and the ICRP Publication 116 data was shown in most frontally positioned organs (e.g., lungs and colon) in AP geometry. The results were consistent with those from a previous study [13], where the testes, colon, and lungs in AP geometry showed less than $10 \%$ difference between 5 Asian voxel phantoms (3 Korean and 2 Japanese phantoms) and ICRP Publication 74 [2]. For 

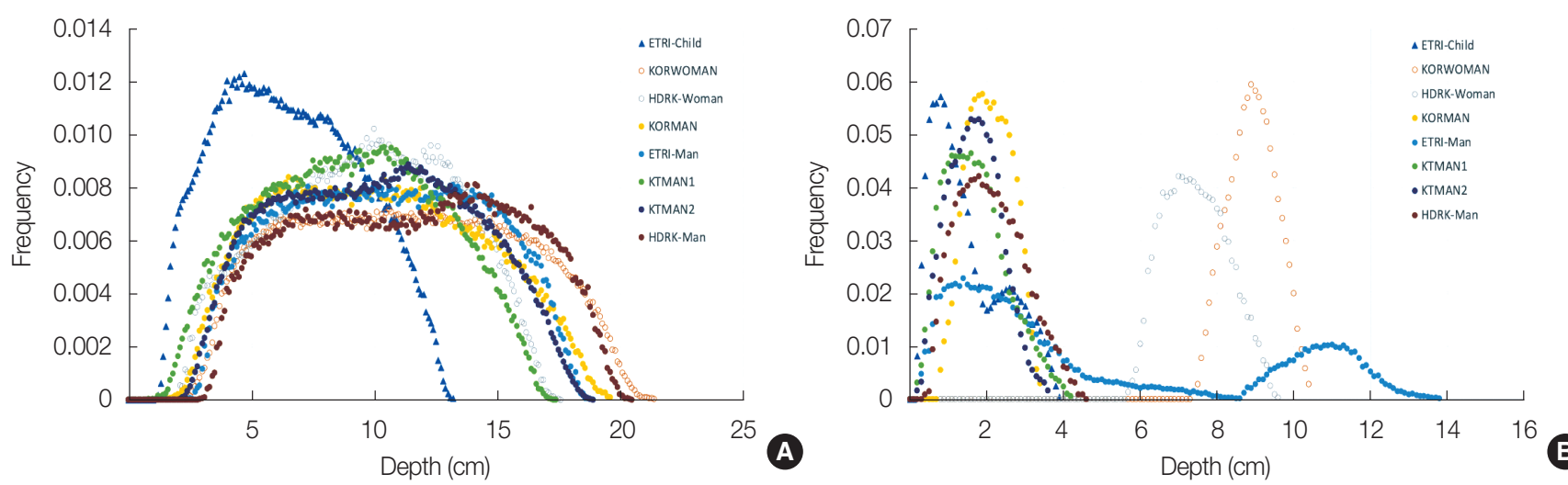

Fig. 5. The depth distribution of lungs (A) and gonads (B) of the 8 Korean phantoms from the phantom surface in anteroposterior geometry.

some deep-seated organs in other irradiation geometries, measurable amounts of variations (e.g., up to $15 \%$ for the female stomach) were observed. However, based on the limited number of Korean phantoms used in the current study, it may not be possible to confirm if the discrepancies are caused by potential anatomical differences between Korean and Caucasian populations.

The authors acknowledge the following limitations in the current study. Not all radiosensitive organs were available for dose calculations in the 8 Korean voxel phantoms. Some radiosensitive organs such as the pancreas and eye lens were not segmented in ETRI Child and ETRI Man because those organs are not of interest in non-ionizing radiation dosimetry. Red bone marrow dose could not be calculated because skeleton segmentation was different across the phantoms: whole skeleton versus separation of cortical and trabecular bones. Lastly, only 8 phantoms may not be enough samples of the Korean population to evaluate the applicability of the ICRP reference data to Korean population.

\section{Conclusion}

A library of DCCs for 13 major radiosensitive organs in a series of pediatric and adult Korean voxel phantoms was established and compared with the reference data from the ICRP. We observed agreement in DCCs in some shallowseated organs but discrepancy in deep-seated organs across the 8 Korean phantoms. Comparison with the data from the ICRP reference adult voxel phantoms showed that our Korean phantom-based data is overall in a reasonable agreement with the ICRP reference data. Further analysis with more Korean-specific computational phantoms may help establish Korean population-specific DCCs.

\section{Conflict of Interest}

No potential conflict of interest relevant to this article was reported.

\section{Acknowledgements}

This research was funded by the intramural research program of the Division of Cancer Epidemiology and Genetics, National Cancer Institute, National Institutes of Health. The views of the authors do not necessarily reflect those of the government. This research was also funded by the MSIP (Ministry of Science, ICT \& Future Planning), Korea in the ICT R\&D Program 2013. The dose calculations utilized the computational resources of the NIH high-performance computing Biowulf cluster (http://biowulf.nih.gov).

\section{Supplementary Materials}

Data files can be found via https://doi.org/10.14407/jrpr. 2020.45.2.69.

\section{References}

1. Petoussi-Henss N, Bolch WE, Eckerman KF, Endo A, Hertel N, Hunt J, et al. ICRP Publication 116. Conversion coefficients for radiological protection quantities for external radiation exposures. Ann ICRP. 2010;40:1-257.

2. Conversion coefficients for use in radiological protection against external radiation. Adopted by the ICRP and ICRU in September 1995. Ann ICRP. 1996;26:1-205.

3. Menzel HG, Clement C, DeLuca P. ICRP Publication 110. Realistic reference phantoms: an ICRP/ICRU joint effort. A report of adult reference computational phantoms. Ann ICRP. 2009;39: 


\section{1-164.}

4. Lee C, Lee J, Lee C. Korean adult male voxel model KORMAN segmented from magnetic resonance images. Med Phys. 2004; 31:1017-1022.

5. Lee CS. Construction of Korean adult voxel phantoms for radiation dosimetry and their applications [dissertation]. Seoul, Korea: Hanyang University; 2002.

6. Lee C, Lee C, Park SH, Lee JK. Development of the two Korean adult tomographic computational phantoms for organ dosimetry. Med Phys. 2006;33:380-390.

7. Kim CH, Choi SH, Jeong JH, Lee C, Chung MS. HDRK-Man: a whole-body voxel model based on high-resolution color slice images of a Korean adult male cadaver. Phys Med Biol. 2008;53: 4093-4106.

8. Yeom YS, Jeong JH, Kim CH, Han MC, Ham BK, Cho KW, et al. HDRK-Woman: whole-body voxel model based on high-resolution color slice images of Korean adult female cadaver. Phys Med Biol. 2014;59:3969-3984.
9. Park JS, Chung MS, Hwang SB, Lee YS, Har DH, Park HS. Visible Korean human: improved serially sectioned images of the entire body. IEEE Trans Med Imaging. 2005;24:352-360.

10. Park S, Lee JK, Kim JI, Lee YJ, Lim YK, Kim CS, et al. In vivo organ mass of Korean adults obtained from whole-body magnetic resonance data. Radiat Prot Dosimetry. 2006;118:275-279.

11. ICRP Publication 89. Basic anatomical and physiological data for use in radiological protection: reference values. A report of age- and gender-related differences in the anatomical and physiological characteristics of reference individuals. Ann ICRP. 2002; 32:5-265.

12. ICRP Publication 103. The 2007 Recommendations of the International Commission on Radiological Protection. Ann ICRP. 2007;37:1-332.

13. Lee C, Lee C, Lee JK. Applicability of dose conversion coefficients of ICRP 74 to Asian adult males: Monte Carlo simulation study. Appl Radiat Isot. 2007;65:593-598. 